hep-th/9704176

April 1997

\title{
Screening in three-dimensional QED
}

\author{
E. Abdalla ${ }^{a}$ and R. Banerjee ${ }^{b \dagger}$ \\ ${ }^{a}$ Instituto de Física-USP, \\ C.P.66318, 05315-970 S. Paulo, Brazil \\ ${ }^{b}$ Instituto de Fúsica da Universidade Federal do Rio de Janeiro \\ CP 68.528, 21.945-970 Rio de Janeiro, Brazil \\ a eabdalla@fma1.if.usp.br \\ ${ }^{b}$ rabin@if.ufrj.br
}

\begin{abstract}
We compute the quark-antiquark potential in three dimensional massive Quantum Electrodynamics. The result indicates that screening prevails for large quark masses, contrary to the classical expectations. The classical result is reproduced for small separation of the quarks.
\end{abstract}

\footnotetext{
${ }^{1}$ On leave of absence from

S.N. Bose National Centre for Basic Sciences

Block JD, Sector III, Salt Lake City, Calcutta 700.091 India
} 
A proper study of the problem of screening and confinement is of considerable importance in our understanding of gauge theories. To avoid the complexities of four dimensions these studies are usually confined to lower dimensions. In this framework, a deep physical interpretation has been achieved. Indeed, in two-dimensional QED[1], one obtains screening in the massless case, but confinement in the massive quark case, realizing the expected picture.

For QCD in two dimensions Gross et al [2] were the first to discuss the subject. If dynamical fermions and test charges are in different representations, they find screening or confinement in some particular cases depending on whether the fermion is massless or massive. A similar conclusion in an identical setting has been arrived at for the massless case in [3]. If, on the other hand, all fermions are in the fundamental representation, then screening prevails independently of the quark mass 迎].

General inquires in two dimensional gauge theories have been performed recently by several authors [5], concerning the $\theta$ vacuum structure, screening, confinement and chiral condensates. In three dimensionons related questions were studied in 6].

It is thus important and instructive to verify how far such issues are just low dimensional unphysical features, or part of the theoretical structure of gauge theories. Usually, the probe of confinement comes from the Wilson criteria, computing the Wilson loop and checking whether it behaves as the area or the perimeter of the loop, for large loops [7]. This is also the approach followed by [2]. Here we follow an alternative route based on the direct calculation of the quark-antiquark potential. To perform the computations we shall take recourse to bosonisation. This is a well known technique in twodimensional space-time [8] which has been well illustrated in getting the Schwinger terms in the current algebra in fermionic field theories, and in order to study the problem of screening and confinement in $\mathrm{QED}_{2}$ [1] as well as in $\mathrm{QCD}_{2}$ [目]. This is possible because one is led to effective actions which contain quantum effects already at the classical level.

The familiar ideas of two dimensional bosonisation have been recently extended to higher dimensions and, in particular, a bosonised form for massive $\mathrm{QED}_{3}$ has been developed 9]. We use this formulation to investigate the phenomenon of screening and confinement in this theory by explicitly computing the quark-antiquark potential. 
The result shows that contrary to the classical expectation, there is screening for large quark mass. However the classical result is reproduced for a small separation of the quarks.

The partition function of three dimensional massive QED in the covariant gauge, in the presence of an external source $J^{\mu}$, is given by

$$
\begin{aligned}
Z= & \int d\left[\psi, \bar{\psi}, A_{\mu}\right] \delta\left(\partial_{\mu} A^{\mu}\right) \exp i \int d^{3} x(\bar{\psi}(i \not \partial-m-e A) \psi \\
& \left.-\frac{1}{4} F_{\mu \nu}^{2}+A_{\mu} J^{\mu}\right)
\end{aligned}
$$

where $F_{\mu \nu}$ is the field tensor, $F_{\mu \nu}=\partial_{\mu} A_{\nu}-\partial_{\nu} A_{\mu}$.

The bosonised version of the above defined action in the large mass limit is given by the expression [9]

$$
\begin{aligned}
Z= & \int d A_{\mu} \delta\left(\partial_{\mu} A^{\mu}\right) \exp i \int d^{3} x\left[-\frac{e^{2}}{8 \pi} \epsilon_{\mu \nu \rho} A^{\mu} \partial^{\nu} A^{\rho}\right. \\
& \left.+\left(\frac{e^{2}}{24 \pi m}-\frac{1}{4}\right) F_{\mu \nu}^{2}+A_{\mu} J^{\mu}+\ldots . .\right]
\end{aligned}
$$

where terms up to order $1 / m$ have been retained in the computation of the effective action as a power series in the inverse quark mass. This result is just the partition function of the Maxwell-Chern-Simons [10] theory in the covariant gauge.

We now compute the potential as being the difference between the Hamiltonian with and without a pair of static external charges separated by a distance $L$, so that

$$
\begin{aligned}
V(L) & =H_{q}-H_{0}=-\left(L_{q}-L_{0}\right) \\
& =-q \int d^{2} x A_{\mu} \delta^{\mu 0}\left[\delta\left(x^{1}+L / 2\right) \delta\left(x^{2}\right)-\delta\left(x^{1}-L / 2\right) \delta\left(x^{2}\right)\right] \\
& =-q\left[A_{0}\left(x^{1}=-L / 2, x^{2}=0\right)-A_{0}\left(x^{1}=L / 2, x^{2}=0\right)\right] .(3)
\end{aligned}
$$

where we have integrated over the two space components in order to find the potential, and considered the source as corresponding to two fixed charges of magnitude $q$ located at the points defined by the respective delta functions. Note that $L_{q}\left(L_{0}\right)$ denote the Lagrangeans in the presence (absence) of the charges.

We now consider the equations of motion associated with the Lagrangean defined in 2. The field equation in the covariant gauge reads

$$
-\frac{e^{2}}{4 \pi} \epsilon_{\mu \nu \rho} \partial^{\nu} A^{\rho}+\left(1-\frac{e^{2}}{6 \pi m}\right) \square A_{\mu}+J_{\mu}=0
$$


Defining the curl of $A_{\mu}$ as

$$
\mathcal{A}_{\mu}=-\epsilon_{\mu \nu \beta} \partial^{\nu} A^{\beta}
$$

the equation of motion can be expressed as

$$
\left[\square+m_{A}^{2}\right] \mathcal{A}_{\mu}=-\frac{1}{1-\frac{e^{2}}{6 \pi m}} \epsilon_{\mu \nu \beta} \partial^{\beta} J^{\nu}-\frac{\frac{e^{2}}{4 \pi}}{\left\{1-\frac{e^{2}}{6 \pi m}\right\}^{2}} J_{\mu}
$$

where $m_{A}=\frac{\frac{e^{2}}{4 \pi}}{\left\{1-\frac{e^{2}}{6 \pi m}\right\}}$. In the absence of sources it reproduces the familiar massive mode of Maxwell-Chern-Simons theory[10]. From (3) it is seen that an expression for $A_{0}$ is required to calculate the potential. This is given in terms of the curl (5) by

$$
\mathcal{A}_{2}=-\partial_{1} A_{0}
$$

The time independent solution for $\mathcal{A}_{2}$ corresponding to the sources describing static quarks can be obtained from (6). Using this result with (7) finally yields

$$
\begin{aligned}
A_{0}(x) & =-\int d^{2} y \Delta\left(x-y, m_{A}\right) \frac{1}{1-\frac{e^{2}}{6 \pi m}} J_{0}(y) \\
& =-\frac{q}{1-\frac{e^{2}}{6 \pi m}}\left[\Delta\left(x^{1}+\frac{L}{2}, x^{2} ; m_{A}\right)-\Delta\left(x^{1}-\frac{L}{2}, x^{2} ; m_{A}\right)\right]
\end{aligned}
$$

where $\Delta\left(x ; m_{A}\right)$ is the Euclidean Feynman propagator in two dimensions, since we are dealing with the time independent Greens function in (6). It is given by the modified Bessel function,

$$
\Delta(x ; M)=\frac{1}{2 \pi} K_{0}\left(M \sqrt{\left(x^{1}\right)^{2}+\left(x^{2}\right)^{2}}\right)
$$

The above solution (8) is defined up to an $x^{2}$ dependent constant, which will be overlooked in what follows, since it will not affect our results.

The potential is now found from (3) and 8 , reading

$$
V(L)=2 \frac{q^{2}}{1-\frac{e^{2}}{6 \pi m}}\left[\Delta\left(0,0 ; m_{A}\right)-\Delta\left(L, 0 ; m_{A}\right)\right]
$$


At this point we disregard the constant term, as discussed before, arriving at the main result of this work,

$$
V(L)=-\frac{1}{\pi} \frac{q^{2}}{1-\frac{e^{2}}{6 \pi m}} K_{0}\left(m_{A} L\right) \equiv-\frac{q_{r e n}^{2}}{\pi} K_{0}\left(m_{A} L\right)
$$

The behaviour of this potential energy should be compared with the classical expectation, that is

$$
V^{\text {class }}(L)=\frac{q^{2}}{\pi} \ln \left(m_{A} L\right)
$$

where we arbitrarily choose the massive parameter. The classical result in the ultraviolet regime corresponds to a renormalisation of the strength, but in the infrared, a logarithmic growth is expected, which would signalize confinement of the external quarks. However, as in the case of two-dimensional QCD the quantum result (11) indicates screening, due to the Chern-Simons term in the action, which induces a mass for the gauge field. Interestingly, in the limit $L \rightarrow 0$ (i.e. when the quarks are close), the leading term in the expression (11) for heavy quarks reduces to the classical result (12). In the two-dimensional case, this is also true, e.g. the classical potential for short separations is given by the expression

$$
V^{\text {class }}(L)=\frac{e^{2}}{2} L
$$

which corresponds to the short separation limit (for heavy quarks) of the full quantum result 1, 国.

In the three dimensional case, we drew the diagrams corresponding to the classical and to the quantum results superposed in figure [1] for comparison.

We see therefore that the conclusions obtained in two-dimensional space-time are valid in the three dimensional case, strengthening them, and providing further reality to the results. The utility of the bosonisation methods in the present context has been clearly illuminated. These methods prove to be of greater effectiveness in obtaining physical results, especially due to the reason that the bosonised version contains quantum corrections at the classical level.

To put our work in the proper perspective we recall that screening effects in the Maxwell-Chern-Simons theory may have been known 
[10] but the observation that there exists a direct connection between fermionic three dimensional QED and the Maxwell-Chern-Simons theory leading to similar effects in the former is new, as shown in our work. Moreover we gave an explicit calculation for the quark-antiquark potential for large values of the quark mass which quantitatively illuminated the screening phenomenon due to the quantum effects. Indeed the departure of the quantum result from the classical expression was clearly illustrated (see figure). It is also appropriate to mention that the results obtained here were based on bosonisation in the large mass limit using the quadratic approximation. It is of course possible to extend the bosonisation scheme to arbitrary mass [11] or to go beyond the quadratic approximation. It would then be interesting to see whether the screening phase persists or is modified under these circumstances. As a concluding remark we mention that screening effects in $\mathrm{QED}_{3}$ have not been investigated either using bosonisation or any other method.

Acknowledgements: this work has been partially supported by Conselho Nacional de Desenvolvimento Científico e Tecnológico, CNPq, Brazil, and Fundação de Amparo à Pesquisa do Estado de São Paulo (FAPESP), São Paulo, Brazil. R.B. thanks the physics department of the UFRJ and USP for the kind hospitality.

\section{References}

[1] H. J. Rothe, K. D. Rothe and J. A. Swieca, Phys. Rev. D19 (1979) 3020.

[2] D. J. Gross, I. R. Klebanov, A. V. Matytsin and A. V. Smilga, Nucl. Phys. B461 (1996) 109, hep-th/9511104.

[3] Y. Frishman and J. Sonnenschein hepth/9701140.

[4] E. Abdalla, R. Mohayaee and A. Zadra Int. J. Mod. Phys $\mathbf{A 1 2}$ (1997) 4539-4557, hepth/9604063. 
[5] G. Grignani, L. Paniak, G.W. Semenoff, hep-th/9705102, L.D. Paniak, G.W. Semenoff, A.R. Zhitnitsky hep-ph/ 9701270, Lori D. Paniak, Gordon W. Semenoff, Ariel R. Zhitnitsky Nucl. Phys. B487 (1997) 191-206 G. Grignani, G. Semenoff, P. Sodano, O. Tirkkonen Int. J. Mod. Phys. A11 4103-4128,1996; A. Zhitnitsky Phys. Rev. D53 (1996) 5821-5833; I. Kogan and A. Zhitnitsky Nucl. Phys. B465 (1996)99-116; B. Chibisov and A. R. Zhitnitsky Phys. Lett. B362 (1995) 105-112.

[6] G. Grignani, G. Semenoff, P. Sodano, O. Tirkkonen Nucl. Phys. B473 (1996) 143-172; A.R. Zhitnitsky Phys. Lett. B291 (1992) 465-472.

[7] K. Wilson, Phys. Rev. 179 (1969) 1499.

[8] S. Coleman Phys. Rev. D11 (1975) 2088; S. Mandelstam Phys. Rev. D11 (1975) 3026.

[9] R. Banerjee Nucl. Phys. B465 (1996) 157.

[10] S. Deser, R. Jackiw and S. Templeton Ann. of Phys. 140 (1982) 372.

[11] R. Banerjee and E.C. Marino, hep-th/9707100, to appear in Nucl. Phys. B 


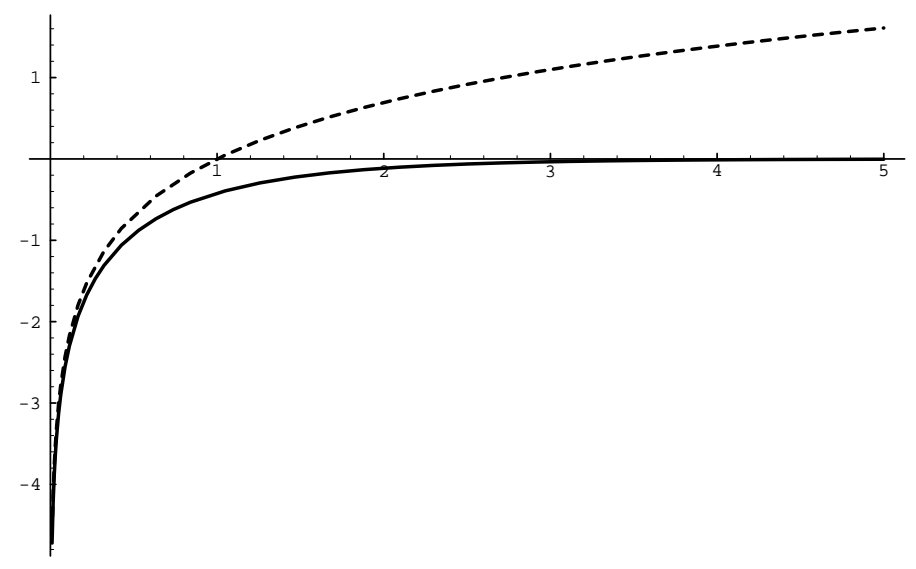

Figure 1: Classical (dashed line) and quantum (continuous line) effective inter-quark potentials are sketched as a function of their separation. The classical potential grows with inter-quark separation (confinement) while for the quantum theory, the potential tends asymptotically to zero. 